\title{
Xiao-Ping Chen-A leading figure among Chinese surgeons
}

Xiao-Ping Chen, male, born in Funan County of China's eastern Anhui Province in 1953, was considered a reformer and innovator in surgery of the liver, pancreas and biliary system. Through four decades of research and clinical work, he has made remarkable achievements in regards to the treatment of hepato-pancreato-biliary diseases and has been elected as a member of the Chinese Academy of Sciences in 2015 because of his outstanding contributions.

Graduated from Bengbu Medical College in 1973, Chen continued to pursue his Master's and Doctor's degree in Surgery from Wuhan Medical College (now Tongji Medical College of Huazhong University of Science and Technology) in 1982 and 1985, respectively. During that period, Chen was under the instruction of two renowned mentors, one is Zaide $\mathrm{Wu}$, the other, Fazu Qiu, a pioneering surgeon from China and member of the Chinese Academy of Sciences, both of whom have a far-reaching influence on Chen's professional growth. Two of Qiu's teachings, as Chen said, guide his research and clinical work all the way: "Be a good person, do good deeds and conduct good research" and "to be a good surgeon, one must operate well, speak well, and write well." In 1986-1987, Chen completed his postdoctoral training under the supervision of Christian Herfarth at Heidelberg University's Department of Surgery. Later, he accepted the offer from University of Cincinnati to join the Department of Surgery, mainly committed to the research on the pathophysiology of acute liver diseases.

Among all the extraordinary achievements Chen has made, modification and improvement of liver transplantation procedure is no doubt the most representative one. Liver transplantation is the only treatment available to some patients who have developed end-stage liver disease. In China back to the early 1980s, due to the severe shortage of organ donors and the low success rate of the operation-about $10 \%$-the treatment remained rather low nationwide. Driven by the urgency of saving lives, Chen did tremendous experimental work and finally, in 1983, the first animal model for auxiliary partial orthotopic liver transplantation was successfully developed. The unappalled procedure not only solved the problem of donor shortage,

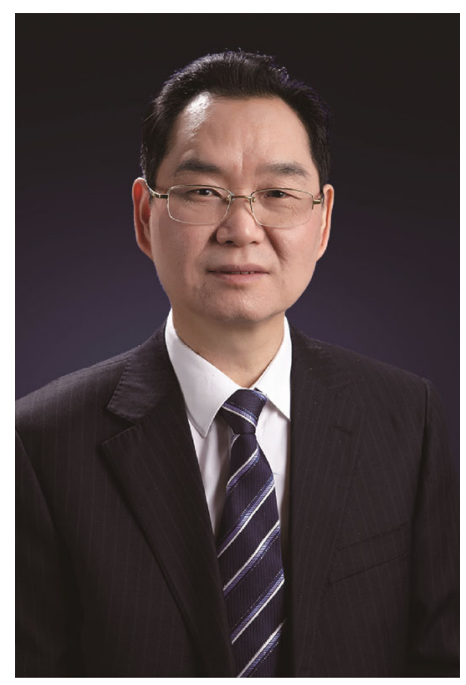

but also overcame the primary reason for the operation failure-the space limitation. Later, more and more clinical applications show that the procedure is safe, effective and feasible.

Moreover, several great practices and techniques have been brought in by Chen in the area of liver cancer. With around 740,000 new cases diagnosed each year in China, $70 \%$ of the tumors in patients diagnosed with hepatocellular carcinoma are categorized as either large (5 to $10 \mathrm{~cm}$ in diameter) or huge (larger than $10 \mathrm{~cm}$ in diameter), which is commonly believed unable to be removed in the medical community. However, in the 1980s, Chen boldly made the hypothesis that though it remained difficult for the operation in clinical practice, the procedure was feasible in theory. To turn the theory into reality, the principal difficulty in front of Chen is intraoperative massive haemorrhage. Three techniques to control bleeding during liver resection was invented: tying up of inflow and outflow vessels without dissecting the hilus of the liver; occlusion of the liver's portal triad and infrahepatic inferior vena cava; and implementation of the liver double-hanging manoeuvre through the retrohepatic avascular tunnel on the right side of the inferior vena cava. By employing the above techniques, intraopera- 
tive blood loss drastically reduces to around $250 \mathrm{~mL}$ from $2000 \mathrm{~mL}$, and accordingly, the mortality rate resulting from the blood loss sharply drops from $10 \%$ to $0.7 \%$.

In the biliary systems, however, it is well known that hilar cholangiocarcinoma is the most common form of cancer affecting the biliary tract. Two major problems exist in the treatment of the disease. Firstly, the conventional choice of operation is hemihepatectomy with caudate lobectomy or extended hemihepatectomy, which involves $60 \%$ to $80 \%$ resection of the liver thus inevitably causing the postoperative complications and high mortality rate. Chen reassessed the efficient and feasible dissection range dimension through numerous of clinical studies and finally confined it to the optimized $20 \%$. The latter problem is the reconstruction of the biliary tract, especially when several bile ducts need to be anastomosed. Combining rich experience and solid theoretical foundation, Chen established an inserting biliary-enteric anastomosis technique, and later, improved this technique by leaving out the stitches of anterior wall of the bile ducts - a useful and critical modification for such a kind complex surgery.

With so many innovations and contributions in the field of hepatobiliary system, Chen, nevertheless, continued to devote himself to the pancreas diseases. Pancreatic head carcinoma is the most complicated as well as the most malignant tumors among all the digestive system neoplasm. Surgical procedure called pancreatoduodenectomy is the most effective way of treatment. However, the critical step-pancreaticojejunostomy-of this surgery involves time-consuming and complex stitching. Moreover, the procedure could induce the high complication rate. Correspondingly, Chen established a procedure described as invaginated pancreaticojejunostomy with transpancreatic U-sutures. The novel and audacious innovation proved to be a breakthrough for pancretoduodenectomy and pancreaticojejunostomy for it only required 2-4 stitches yet the pancreatic stump automatically inserted to the intestinal lumen when pulling the stitches. It is no exaggeration to say that no other procedure ever developed can be so time-saving as well as effective.

Live and learn. Chen is without doubt the proper and exact interpretation of the old saying. For over 40 years of devotion and dedications in the career of surgeon, Chen broke with convention over and over again and promoted the hepato-pancreato-biliary surgery to a new level in China. Great respect as well as honor has been unsparingly given to him from communities of surgeons around the world. An honorary fellow of the American Surgical Association since 2011, an honorary professor of the University of Insubria in Italy since 2013, executive council member of the International Hepato-Pancreato-Biliary Association (IHPBA) and the president of the Chinese Chapter of the IHPBA, he has also been appointed as the president of A-PHPBA since 2013. Undoubtedly, Chen is a fullydeserving leading figure of reform and innovation in surgery of the liver, pancreas and biliary system.

Open Access This article is distributed under the terms of the Creative Commons Attribution License which permits any use, distribution, and reproduction in any medium, provided the original author(s) and source are credited. 